\title{
Using Mobile Systems to Monitor an Ambulatory Patient
}

\author{
Ângelo Costa, Guilherme Barbosa, Tiago Melo, and Paulo Novais
}

\begin{abstract}
Medical diagnostics and vital signs monitoring demands more technological solutions to cope with new methods of treatment. Continuous monitoring and information processing tools are vital to a physician with several patients under his care. In this work, a system that relies on agents and mobile and wireless devices is presented. Its use with small scale sensors allows to collect and analyse vital data in real-time, triggering appropriate reactions in case of eminent danger. This includes real-time notifications to practitioners. In cases in which the physician is unable to divide his attention among all his patients, the system is able to drive his attention to one patient only and, when it is necessary, to another one, according to their medical state. This concept represents a breakthrough in terms of the physician's time and task management, being possible to apply it two major scenarios: patient recovery in a hospital environment or elderly living alone in a domestic environment. In that sense, we present a brief contextualiation of the problem as well as the architecture and technologies used to implement the proposed work.
\end{abstract}

\section{Introduction}

The increase in the quality of health care services, along with socio-economic growth and technological achievements are making life expectancy higher. Moreover, the latest United Nations (UN) reports on world population [1] show the current population ageing.

Due to the overall growth in the elderly population, constant monitoring of some specific groups of population has become increasingly necessary, namely in groups that suffer from chronic diseases and heart conditions. Although transport availability has increased over the years, frequent travel to health care facilities is not a viable

CCTC, Departamento de Informática, Universidade do Minho, Braga - Portugal e-mail: acosta@di.uminho.pt,gbarbosa@gmail.com, tiago.blackcode@gmail.com, pjon@di.uminho.pt 
solution, mainly in cases that need continuous care. Moreover, this can also have a negative impact on the patient's quality of life.

With the technological progress in the fields of electronics and computing, wearable and environmental sensors have become more compact and portable, while providing high precision measurements of the parameters they are sensing. This allows to develop applications that explore sensor's capabilities in mobile and portable devices that a patient can carry, without being intrusive or affecting the person's daily life.

Based on these ideas, we propose a portable monitoring system, implemented over a mobile device (e.g. PDA, smart-phone), capable of transmitting information collected by sensors worn by the patient to an external medical entity, which in turn will generate a medical diagnosis of the patient being monitored. In this case, the focus is on constant vital sign monitoring in order to anticipate heart failure. With these goals in mind, this system allows practitioners to provide instant and localized health-care services to those being monitored and avoid critical scenarios which can ultimately lead, in an unsupervised environment, to the patients' death.

\subsection{Related Projects}

Some projects exist that already have working devices and systems. Their development is important and allows to compare the features and, if proven useful, use the hardware and underlaying platforms to develop new applications with different purposes.

Vital Jacket [3] is a vital sign monitoring system integrated in the patient's clothing, combining both textile and micro-electronic components. VitalJacket is composed of a t-shirt that accommodates a small, lightweight and compact electronic device that constantly monitors the user's heartbeat.The electronic device has an integrated Bluetooth module for data transmission.

Plux [2] is a Portuguese company specialized in the design, development and production of systems for data acquisition and wireless sensors to study the electrophysiological activity. The data acquisition systems also communicate with the computing devices via Bluetooth.

\section{2 iNumon- Independent User Monitoring}

This project aims at being an answer to the previously presented problems. The question of on-body monitoring is a very complicated one, including challenges about reliability and choice of correct sources of data. It is also at steak the level of adaptation of the user to the entire system.

In the following pages, we will present our insights and the solutions that we propose in order to achieve the established goal. The project is organized into several 
main modules: the Sensor Network, the mobile devices and the server-side application. These modules are combined to develop a mobile monitoring system that can make the appropriate decisions. In this section we describe the architecture, the technologies, the developed modules and the results observed.

\subsection{System Architecture}

As mentioned before, the system proposed aims at a constant monitoring of relevant information about a patient, relying on the concept of Body Area Network (BAN). BANs will be operating over a mobile device that records the patient's condition through a sensor network and forwards the information collected to an external entity $[12,13]$. A set of sensors must thus capture the specific condition of a patient in a discrete time interval and use such information for remote diagnose, to assess the patient health condition and detect eventual anomalies (Fig. 1).

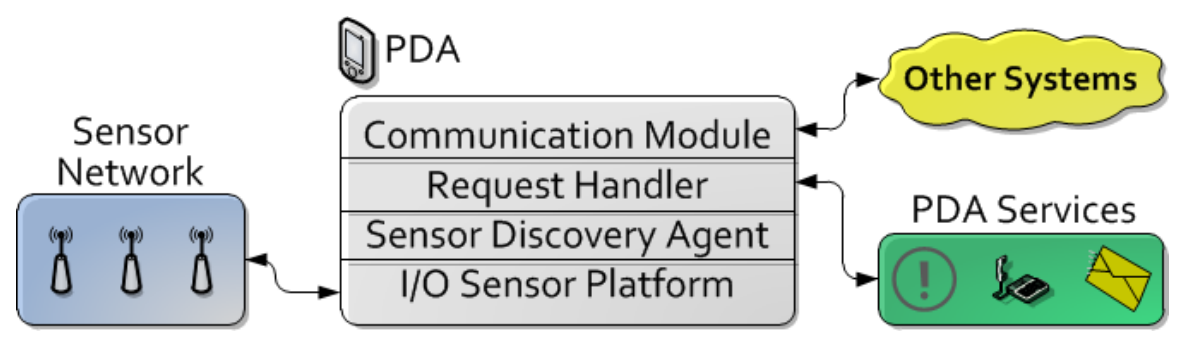

Fig. 1 The architecture of the proposed system.

This sensor network must be flexible enough to allow engaging or disengaging sensors without any kind of system reboot and without affecting the performance and efficiency of the system. Inside the BAN, a set of software agents will also reside, focused on providing interaction services to the remote entities [11]. These are able to process the result of the diagnose within the mobile device, making it possible to inform about the patient's condition after a successful diagnosis based on the information provided by his mobile device in the first place.

\subsubsection{Agents' Characteristics}

The agents that interpret the data provided by the sensors must only retain, manage and forward the information.

The forwarding of the information gathered by the sensors through a network to an external diagnosis entity is done by an agent called Sensor Discovery Agent (SDA) and has management-oriented characteristics. 
These management abilities are associated with basic characteristics that this agent must comply with at the interaction level with the Sensor System Agents (SSA) and the I/O communication module in order to guarantee the existence of a data flow between the sensors and the respective SSA. Therefore, the SDA must receive notifications every time a sensor enters or exits the BAN through the I/O module. This allows the SDA to load or unload the sensor agents according to the system's needs.

Moreover, the SDA must gather information obtained by the SSA and forward it to an external entity so that a diagnosis of the patient's current state can be created.

To carry out information forwarding, a module called Communication Platform (CP) is being used. This module is responsible for the encapsulation of the available information and sending it along the network to the remote entity.

\subsubsection{Communication Technologies}

This project encompasses several methods and technologies of communication. In this section we describe the main technologies that were used to implement the proposed architecture.

In our first tests, the ZigBee technology was used (Figure 2) [5, 6, 7]. We have abandoned ZigBee in favour of Bluetooth. Specifically, the platform of reception was too large and did not support all of the mobile systems.

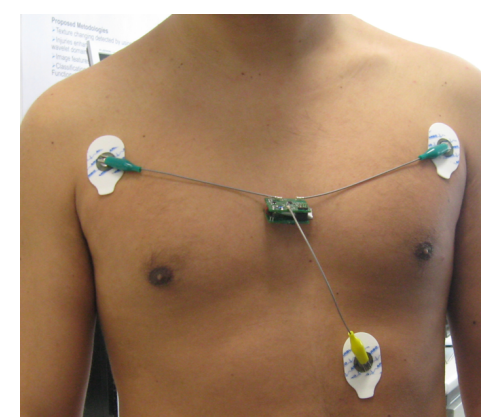

(a) EGC Capture with ZigBee communication.

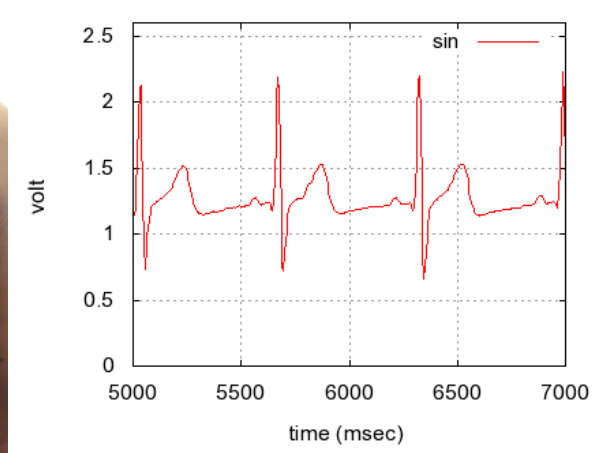

(b) Result of the ECG Capture.

Fig. 2 Representation of the ECG device and the visual representation of the collected data

In terms of agent support and communication, the OSGi Platform is being used [8]. A Multi-agent System (MAS) [9, 10] supported by JADE Framework using FIPA-ACL standard and XML for the message content is our base supporting system to the developed agents. 


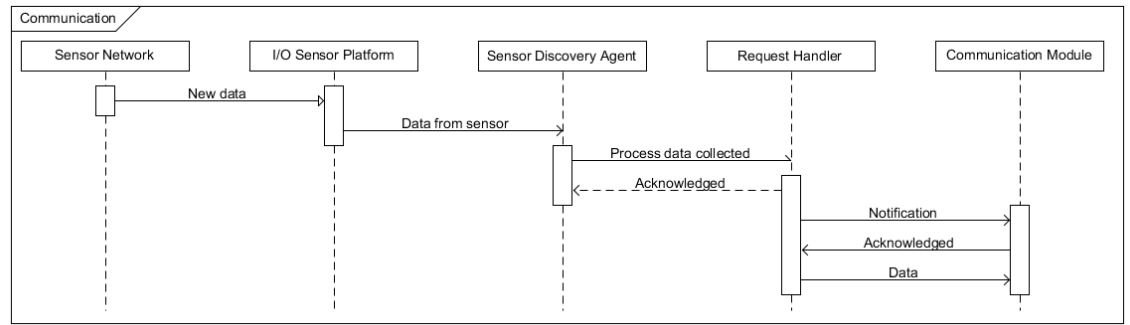

Fig. 3 Typical communication between the Agents and platforms

\subsubsection{Sensor Monitoring}

With the emergence of increasingly compact and precise sensors, the concept of Sensor Monitoring has become viable in the sense that a patient wearing a set of sensors can have a normal daily life without any discomfort or mobility difficulties.This technology is seen as very positive by the medical field as it allows to constantly control and monitor a patient's vital signs with no need for the him to be located inside any medical facility. Within this subject, the goal is to apply the concept of BAN as it is defined above, with some architecture specific variants that promote an intelligent, modular and flexible interaction between the different components.

The main challenge that arises from the use of sensors is the quality of the information received. One of the major problems here is the fact that sensors are not static and the user can, in fact, misuse them or damage them.

To monitor the sensors and measure the quality of the information provided by them, two different operating modules are considered: The first approach dictates that to communicate directly with the system's sensor hardware a low-level communication module is necessary. The module can be seen as a set of small software components that interact with a specific sensor and depend on the technology that the sensor uses to communicate with the monitoring device.

The second approach dictates that the agent-oriented platform must be able to read and filter the data coming through the sensors at a higher level. The agents that form this platform operate independently of the technology used by the sensors, which justifies the use of the first module mentioned before.

Every agent communicates directly with the respective software component that handles low-level communication to establish an information bridge and allow the agent to read and interpret the information.

The I/O Sensor Platform is thus responsible for encapsulating the different communication methods and protocols and will inform the system when a sensor enters or exits the BAN.

In Figure 4, a photo of the application running in a PDA is depicted. The information is from a live feed of the sensor platform and it is being processed in real-time. The data collected can be represented as the graph presented in the screen shows. 


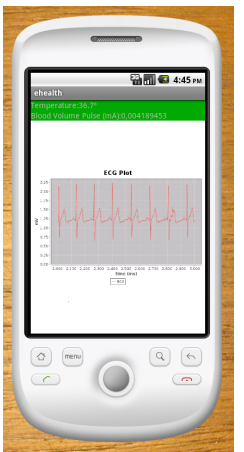

(a) Execution on Mobile Device.

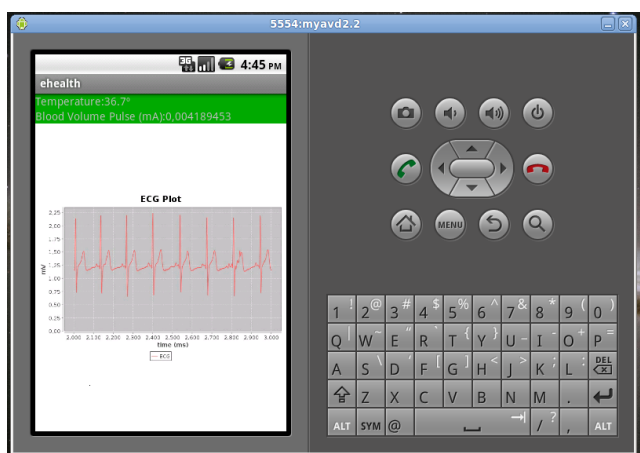

(b) Development interface.

Fig. 4 Two representations of the Advanced User Interface.

Currently we are testing the implementation of a portable ECG sensor platform in this system, which is reliable and more compact when compared with classical ECG sensors.

\subsection{User Notification}

Besides the monitoring component of the system, it is also convenient that the user of the device can be informed about his/her health condition. Moreover, location-based services can also be interesting in cases of emergency. This is implemented using several approaches, ranging from a notification to the user about his state using the interface of the application or audio alerts.With this level of flexibility, it is possible for a medical practitioner to fully support a patient regardless of his/her consciousness state.

In the case of a user alert operation, the agent responsible for delivering this kind of information has a set of pre-determined definitions so that it can provide the user with very precise and clear information about his/her di-

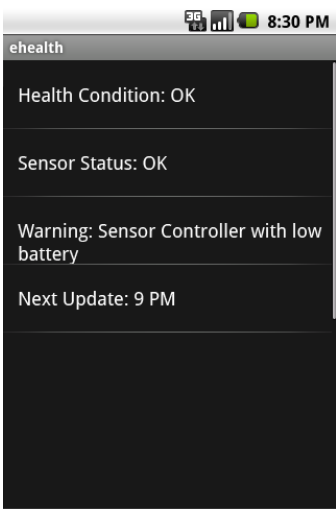

Fig. 5 User Mobile Interface agnosis.

This way, the user receives notifications if he/she needs some specific medical procedure concerning his/her clinical condition. On the other hand, if a text message or telephony operation is requested, the appropriate agents must be aware of the people directly involved in the patient's monitoring and therefore provide such configuration. 


\section{Results}

The system presented in this paper essentially implements the collection of information on a ECG sensor, including the values of blood pressure and body temperature. The ECG data was collected by real sensors wore by a test subject. In addition, in order for more data to be available for testing purposes, a simulation platform was developed. This platform emulates data captured by an ECG sensor, following functions extracted from the real temporal series of values. The simulation can emulate both normal and abnormal health states and is running on an Android powered mobile device. The data collected about the user's body temperature and blood pressure value is used the same way as the one generated by the ECG simulation platform.

The development of the Android application and the Server has also the advantage of, by these being modular platforms, allow the services to be developed independently, without having to depend on the development of the sensor platform or any other module. Currently, the work focuses on the combined use of the Android platform and OSGi, with a special regard on the sensor fusion [15] theme.

From our point of view, the simulation platform is a first step that will support the rest of the development process. Its importance is even higher when we consider the risks of developing and testing in real environments, with real patients.

Another major feature is the fact that the project can communicate with other applications and projects in a transparent way. A concrete case is the integration with the iGenda project [12]. This agenda manager deals with the problems of automatic scheduling of events. In this case, the innovative factor is the inclusion of, for instance, the event of scheduling a visit to the physician or call the emergency system in case of an event involving critical readings of sensors or deterioration of the health condition.

\section{Conclusion}

The system presented in this paper covers different users (patients, physicians) and the use of a mobile device to capture the data and pre-process it, gives more mobility to the user, not interfering with his daily life. It is our conviction that the community has a lot to benefit from this project as it will improve the quality of life of people in need of constant health care.

Moreover, this project may indirectly relieve already congested medical institutions and services. Given the modular architecture of the system mostly relying on standard protocols of communication, the integration with other projects is possible, allowing to develop a wide range of services, focused on very different fields.

In future work we aim to explore a possible architecture for a complementary system to the mobile architecture presented here, which can achieve automatic and intelligent diagnose based on the information gathered. Moreover, another future goal is to provide the mobile device with learning skills This will focus on the interaction of the sensor agents and the data they gather from the sensors, and the user 
notification that will be taking place each time a diagnose is received by the mobile device.

Finally, a note about the use of a mobile device as a type of web-service with a set of properties and operations. This approach can be interesting in many other fields such as tourism, data synchronization or location-aware services.

\section{References}

1. Department of Economic and Social Affairs, Population Division: World Population Ageing. United Nations (2009)

2. Shahriyar Amini, Priya Narasimhan: Twitter Jacket, An automated activity and health monitoring solution for the elderly. (2009)

3. Biodevices, S.A http://www.biodevices.pt/ VitalJacket

4. David Malan, Thaddeus Fulford-Jones, Matt Welsh, Steve Moulton: CodeBlue: An Ad Hoc Sensor Network Infrastructure for Emergency Medical Care. MobiSys 2004 Workshop on Applications of Mobile Embedded Systems, (2004)

5. William Lehr, Lee W. McKnight: Wireless Internet access: $3 \mathrm{G}$ vs. WiFi. Telecommunications Policy, Volume 27, Issues 5-6, (2003)

6. Roger M. Whitaker, Leigh Hodge, Imrich Chlamtac: Bluetooth scatternet formation: A survey. Ad Hoc Networks, Volume 3, Issue 4, (2005)

7. D. Gislason: Zigbee Wireless Networking. Newnes, (2008)

8. Hsuan-Yu Huang, Wei-Chung Teng, and Sheng-Luen Chung: Smart Home at a Finger Tip: OSGi-based MyHome Proceedings of the 2009 IEEE International Conference on Systems, Man, and Cybernetics, (2009)

9. Sycara K.: Multiagent Systems. Artificial Intelligence Magazine, (1998)

10. Jamshid Bagherzadeh, S. Arun-Kumar: Flexible Communication of Agents based on FIPAACL. Electronic Notes in Theoretical Computer Science, Volume 159, (2006)

11. Benoît Latré and Bart Braem and Chris Blondia and Ingrid Moerman and Piet Demeester: A Survey on Wireless Body Area Networks Wireless Networks, Springer, (2010)

12. Costa A., Novais P., Costa R., Corchado J.M., Neves J., Multi-Agent Personal Memory Assistant, in Trends in Practical Applications of Agents and Multiagent Systems, Yves Demazeau et al. (eds), in Advances in Intelligent and Soft Computing, Vol. 71, Springer- Verlag, ISBN 978-3-642-12432-7, pp. 97-104, (PAAMS 10 - The 8th International Conference on Practical Applications of Agents and Multi-Agent Systems, University of Salamanca, Spain, 26-28th April/2010), (2010)

13. Costa R., Novais P., Lima L., Bulas-Cruz J. and Neves J., VirtualECare: Group Support in Collaborative Networks Organizations for Digital Homecare, in Handbook of Digital Homecare, Springer Series: Series in Biomedical Engineering, Yogesan, K.; Bos, L.; Brett, P.; Gibbons, M.C. (Eds.), ISBN: 978-3-642-01386-7, (2009)

14. Óscar Gama, Paulo Carvalho, J. A. Afonso, P. M. Mendes: QoS Deployment in Wireless eHealth and e-Emergency: Main Issues and a Case-study in Proc. 3th Symposium of Ubiquitous Computing and Ambient Intelligence 2008 (UCAmI'08), Advances in Soft Computing Series, Springer-Verlag, (2008)

15. Peter R. DeLong: Interoperability \& Sensor Fusion. Naval Engineers Journal, Volume 115, Blackwell Publishing Ltd (2003) 